\title{
EXTINCTION PROBABILITIES OF SUPERCRITICAL DECOMPOSABLE BRANCHING PROCESSES
}

\author{
SOPHIE HAUTPHENNE, ${ }^{*}$ Université Libre de Bruxelles and The University of Melbourne
}

\begin{abstract}
We focus on supercritical decomposable (reducible) multitype branching processes. Types are partitioned into irreducible equivalence classes. In this context, extinction of some classes is possible without the whole process becoming extinct. We derive criteria for the almost-sure extinction of the whole process, as well as of a specific class, conditionally given the class of the initial particle. We give sufficient conditions under which the extinction of a class implies the extinction of another class or of the whole process. Finally, we show that the extinction probability of a specific class is the minimal nonnegative solution of the usual extinction equation but with added constraints.
\end{abstract}

Keywords: Multitype branching process; decomposable branching process; extinction criteria; extinction probability

2010 Mathematics Subject Classification: Primary 60J80

Secondary $60 \mathrm{~J} 10$

\section{Introduction}

Decomposable (or reducible) multitype branching processes differ in several ways from irreducible processes. In the literature, authors generally focus on the asymptotic behaviour of such branching processes without discussing extinction criteria or algorithmic issues.

Let $\{\boldsymbol{Z}(t), t \in \mathbb{N}\}$ denote a supercritical discrete-time multitype branching process, where $\boldsymbol{Z}(t)$ is a row vector whose $i$ th entry is the size of the type-i population at time $t$; let $\rho>1$ be the spectral radius of the mean offspring matrix $\boldsymbol{M}$, and let $\boldsymbol{v}$ be its corresponding positive left eigenvector. In the case where $\boldsymbol{M}$ is irreducible, Kesten and Stigum [8] showed that there exists a one-dimensional random variable $w$ such that $\lim _{t \rightarrow \infty}\left(\boldsymbol{Z}(t) / \rho^{t}\right)=w \boldsymbol{v}$ with probability 1: the eigenvalue $\rho$ plays the role of a normalizing constant; all types grow at the same rate $\rho$. The same authors proved in [9] that in the reducible case, the asymptotic behaviour of $\boldsymbol{Z}(t)$ depends on the type of the initial individual. In addition, different subsets of components of the vector $\boldsymbol{Z}(t)$ have, in general, different normalizing constants: different types may grow at different rates. The corresponding limit vector shows a greater variety of qualitative properties than in the irreducible case.

Foster and Ney [2] studied the asymptotic behaviour of the extinction probability of the various types in a critical multitype decomposable Galton-Watson process. They also emphasized that, unlike in the irreducible case, the asymptotic behaviour depends on the type of the initial particle. In another paper [3], still in the critical case, they analyzed limiting distributions of the population size in different subsets of particle types, normalized by the appropriate constants, and conditioned on existence or nonexistence of some subsets of particle types.

Received 19 November 2010; revision received 16 February 2012.

* Postal address: Department of Mathematics and Statistics, The University of Melbourne, Parkville, VIC 3010, Australia. Email address: sophiemh@unimelb.edu.au 
Among other authors, we also refer the reader to the work of Sugitani [14], who also focused on critical decomposable Galton-Watson processes and described general limit theorems, and to Sewastjanow [13, Chapter 4], who made a classification of the types in a reducible branching process.

From a practical point of view, decomposable branching processes have many applications in biology and epidemiology: Olofsson [11] used a decomposable branching process as a model of telomere shortening, and Scalia-Tomba [12] studied the asymptotic final size distribution of reducible multitype Reed-Frost epidemic processes.

Here, we investigate extinction probabilities of discrete-time supercritical decomposable multitype branching processes. The types are partioned into irreducible equivalence classes. We distinguish among two types of extinction event: the whole process may become extinct, which corresponds to total extinction, and there may also be situations where the process lives forever while some classes become extinct and will never be generated again, which we classify as partial extinction (a precise definition is given later).

We derive necessary and sufficient conditions for total and partial extinctions, as well as for the extinction of a specific class, conditionally given the class of the initial particle. We give sufficient conditions under which the extinction of a class implies the extinction of another class or of the whole process. Finally, we show that the extinction probability of a specific class is the minimal nonnegative solution of the usual extinction equation but with added constraints, and that it is also equal to the total extinction of a modified branching process. We discuss the algorithmic issues to compute the partial extinction probabilities, in particular for special classes of multitype branching processes called Markovian trees [1], [6], [7].

The paper is organized as follows. In Section 2 we recall a fundamental result about the extinction probability of a multitype branching process, and we define partial extinction. In Section 3 we prove various extinction criteria. Finally, in Section 4 we characterize the partial extinction probabilities and discuss computational matters.

\section{Definitions and notation}

Let $\left\{\boldsymbol{Z}(t)=\left(Z_{1}(t), \ldots, Z_{n}(t)\right), t \in \mathbb{N}\right\}$ be a multitype branching process, where $Z_{i}(t)$ represents the number of individuals of type $i$ alive at time $t, 1 \leq i \leq n$. We denote by $P_{i}(s)$ the offspring generating function of an individual of type $i$, that is,

$$
P_{i}(s)=\mathrm{E}\left[s^{Z(1)} \mid \varphi_{0}=i\right] \text { for } s=\left[s_{1}, s_{2}, \ldots, s_{n}\right]^{\top} \text { with }\left|s_{i}\right| \leq 1,
$$

where the notation $\boldsymbol{s}^{Z(1)}$ stands for $s_{1}^{Z_{1}(1)} s_{2}^{Z_{2}(1)} \cdots s_{n}^{Z_{n}(1)}$, and $\varphi_{0}$ is the type of the progenitor: $\varphi_{0}=i$ means $\boldsymbol{Z}(0)=\boldsymbol{e}_{i}$, where $\boldsymbol{e}_{i}$ is a vector whose $i$ th component is 1 and whose other components are all 0 . Here ${ }^{~} \top$, denotes the transpose operator. Accordingly, $\boldsymbol{P}(\boldsymbol{s})=$ $\left[P_{1}(s), P_{2}(s), \ldots, P_{n}(s)\right]^{\top}$ is the offspring generating vector.

We use the notation 1 for the column vector $[1,1, \ldots, 1]^{\top}$, and write $s \leq \boldsymbol{t}$ and $\boldsymbol{s}<\boldsymbol{t}$ for two vectors $s$ and $\boldsymbol{t}$ if $s_{i} \leq t_{i}$ and $s_{i}<t_{i}$, respectively, for all $i$.

The matrix $\boldsymbol{M}=\left(M_{i j}\right)$ represents the mean offspring matrix: $M_{i j}=\left.\left(\partial P_{i}(s) / \partial s_{j}\right)\right|_{s=1}$ is the mean number of children of type $j$ generated by a parent of type $i, 1 \leq i, j \leq n$.

A multitype branching process is said to be irreducible when any type of individual may have individuals of all types among their (direct or indirect) descendants with positive probability. Otherwise, it is called reducible. This is stated more formally as follows.

Definition 2.1. A multitype branching process is irreducible if the mean offspring matrix $\boldsymbol{M}$ is irreducible, that is, if, for each $1 \leq i, j \leq n$, there exists an integer $k \geq 0$ such that $\left(\boldsymbol{M}^{k}\right)_{i j} \neq 0$. 
Definition 2.2. A multitype branching process is singular if the $n$ generating functions $P_{1}(s)$, $\ldots, P_{n}(s)$ are linear in $s_{1}, \ldots, s_{n}$ (thus without constant term), that is, if each individual generates exactly one and only one direct descendant.

Let $\boldsymbol{q}=\left[q_{1}, q_{2}, \ldots, q_{n}\right]^{\top}$ be the vector of conditional extinction probabilities of $\boldsymbol{Z}(t)$, given the type of the initial individual, that is,

$$
q_{i}=\mathrm{P}\left[\text { there exists } T<\infty: \boldsymbol{Z}(T)=\mathbf{0} \mid \varphi_{0}=i\right], \quad 1 \leq i \leq n .
$$

This is a total extinction: there will be no individual alive after time $T$.

Let $\rho=\operatorname{sp}(M)$ be the spectral radius of the matrix $\boldsymbol{M}$. The following theorem is a fundamental result in the theory of branching processes. A nice proof may be found, for instance, in [10, Chapter 1, Theorem 7.1].

Theorem 2.1. Assume that the branching process is nonsingular.

- If $\rho<1$ then $\boldsymbol{q}=\mathbf{1}$, and we say that the branching process is subcritical.

- If $\rho=1$ then $\boldsymbol{q}=\mathbf{1}$, and we say that the branching process is critical.

- If $\rho>1$ then we say that the branching process is supercritical, and $\boldsymbol{q}<\mathbf{1}$ in the irreducible case, $\boldsymbol{q} \leq \mathbf{1}$ with $\boldsymbol{q} \neq \mathbf{1}$ otherwise.

In all cases, $\boldsymbol{q}$ is the minimal nonnegative solution of the vector equation

$$
P(s)=s
$$

in the sense that any other solution $\boldsymbol{x}$ of (2.1) is such that $\boldsymbol{q} \leq \boldsymbol{x}$.

In practice, the extinction probability vector $\boldsymbol{q}$ may be obtained by applying the functional iteration on the extinction equation (2.1) (see [5, Chapter 2, Theorem 7.2]). Let $\boldsymbol{P}^{(1)}(\boldsymbol{s})=\boldsymbol{P}(\boldsymbol{s})$ and $\boldsymbol{P}^{(k)}(\boldsymbol{s})=\boldsymbol{P}\left(\boldsymbol{P}^{(k-1)}(\boldsymbol{s})\right)$ for $k \geq 2$.

Theorem 2.2. If $\mathbf{0} \leq \boldsymbol{a}<\mathbf{1}$ then $\lim _{k \rightarrow \infty} \boldsymbol{P}^{(k)}(\boldsymbol{a})=\boldsymbol{q}$. This still holds if $\boldsymbol{a} \leq \mathbf{1}$ with $\boldsymbol{a} \neq \mathbf{1}$ in the irreducible case.

From now on, we assume that the branching process $\{\boldsymbol{Z}(t), t \in \mathbb{N}\}$ is supercritical and reducible, that is, there exist indices $1 \leq i, j \leq n$ such that, for all $k \geq 0,\left(\boldsymbol{M}^{k}\right)_{i j}=0$; this means that individuals of type $i$ will never have any individual of type $j$ among their descendants.

The matrix $\boldsymbol{M}$ may be written in a normal form, possibly after a permutation of indices (see [4, Chapter 13]):

$$
\boldsymbol{M}=\left[\begin{array}{ccccc}
\mathcal{M}_{11} & 0 & 0 & \cdots & 0 \\
\mathcal{M}_{21} & \mathcal{M}_{22} & 0 & \cdots & 0 \\
\mathcal{M}_{31} & \mathcal{M}_{32} & \mathcal{M}_{33} & \cdots & 0 \\
\vdots & \vdots & \vdots & \ddots & \vdots \\
\mathcal{M}_{m 1} & \mathcal{M}_{m 2} & \mathcal{M}_{m 3} & \cdots & \mathcal{M}_{m m}
\end{array}\right]
$$

where the diagonal blocks $\mathcal{M}_{k k}$ are irreducible or equal to 0 , and the subdiagonal blocks $\mathcal{M}_{k \ell}$ for $k>\ell$ are nonnegative. We write $\mathcal{M}_{k \ell}(1 \leq k, \ell \leq m)$ for the $(k, \ell)$ th submatrix of $\boldsymbol{M}$, in order to distinguish it from $M_{i j}$, which is the $(i, j)$ th component of $\boldsymbol{M}$. 
We will say that there is a path from type $i$ to type $j$, and write $i \rightarrow j$, if there exists a nonnegative integer $n$ such that $\left(\boldsymbol{M}^{n}\right)_{i j}>0$, that is, if an individual of type $i$ has a descendant of type $j$ with positive probability. We will write $i \leftrightarrow j$ if $i \rightarrow j$ and $j \rightarrow i$. The relation ' $\leftrightarrow$ ' is an equivalence relation; accordingly, the $n$ types of branching process $\{\boldsymbol{Z}(t), t \in \mathbb{N}\}$ are partitioned into $m \leq n$ equivalence classes $C_{k}, 1 \leq k \leq m$ (see [10, Section 2.3]). The diagonal blocks of the normal form of the matrix $\boldsymbol{M}$ reflect this partition: $\mathcal{M}_{k k}=\left(M_{i j}\right)_{i, j \in C_{k}}$ and $\mathcal{M}_{k \ell}=\left(M_{i j}\right)_{i \in C_{k}, j \in C_{\ell}}$.

We can extend paths to classes by saying that there is a path from class $C_{k}$ to class $C_{\ell}$, and write $k \rightarrow \ell$ if there are $i \in C_{k}$ and $j \in C_{\ell}$ such that $i \rightarrow j$.

We define the following sets of class indices for $1 \leq k \leq m$ :

$$
\mathscr{D}_{k}=\{\ell \leq k: k \rightarrow \ell\}, \quad \mathcal{A}_{k}=\{\ell \geq k: \ell \rightarrow k\}, \quad \mathcal{E}_{k}=\left\{\ell<k: \mathcal{M}_{k \ell} \neq 0\right\} .
$$

In other words, $\mathscr{D}_{k}$ is the set of classes descending from $C_{k}$ and $\mathscr{A}_{k}$ is the set of ancestor classes of $C_{k}$. Clearly, $\ell \in \mathcal{A}_{k}$ if and only if $k \in \mathscr{D}_{\ell}$. Finally, $\ell \in \mathcal{E}_{k}$ means that an individual in class $C_{k}$ has a positive probability of directly generating an individual in class $C_{\ell}, k \neq \ell$.

For each $k$, if $\varphi_{0}=i$, where $i \in C_{k}$, the subprocess $\left\{\boldsymbol{Z}^{k}(t)=\left[Z_{j}(t)\right], j \in C_{k}, t \in \mathbb{N}\right\}$, restricted to the individuals whose type is in $C_{k}$, is an irreducible branching process with mean offspring matrix $\mathcal{M}_{k k}[9]$.

It is well known [4] that, when a matrix $\boldsymbol{M}$ has the form given by (2.2), its spectrum is precisely the union of the spectra of the $\mathcal{M}_{k k}, 1 \leq k \leq m$, so that the spectral radius of $\boldsymbol{M}$ is $\operatorname{sp}(\boldsymbol{M})=\max _{1 \leq k \leq m} \operatorname{sp}\left(\mathcal{M}_{k k}\right)$. Therefore, one supercritical subprocess $\left\{\boldsymbol{Z}^{k}(t), t \in \mathbb{N}\right\}$ is enough for the whole process $\{\boldsymbol{Z}(t), t \in \mathbb{N}\}$ to be supercritical as well. However, the behaviour of the reducible branching process is not determined by the spectral radius of $\boldsymbol{M}$ only; we shall demonstrate in the next section how the criticality of the different subprocesses $\left\{\boldsymbol{Z}^{k}(t), t \in \mathbb{N}\right\}$ influences the behaviour of the whole process $\boldsymbol{Z}(t)$. Let us first introduce some notation.

Let $E$ denote the total extinction event: $E=\{$ there exists $T>0: \boldsymbol{Z}(T)=\mathbf{0}\}$. We write $\boldsymbol{q}_{k}$ for the vector of extinction probability of the whole process, given that the initial individual belongs to class $C_{k}$. That is, for each $i \in C_{k}$, the $i$ th entry of $\boldsymbol{q}_{k}$ is given by

$$
\left(\boldsymbol{q}_{k}\right)_{i}=\mathrm{P}\left[E \mid \varphi_{0}=i\right] .
$$

With this, $\boldsymbol{q}=\left[\boldsymbol{q}_{1}, \boldsymbol{q}_{2}, \ldots, \boldsymbol{q}_{m}\right]^{\top}$ denotes the vector of conditional extinction probabilities of the whole process, given the class of the initial individual.

Now observe that if we restrict our attention to the class $C_{\ell}$ only, then the event $\{$ there exists $T>0: Z^{\ell}(T)=\mathbf{0}$ \} does not necessarily imply the extinction of class $C_{\ell}$ since at time $T$, some individual in another class might be alive and able to produce new individuals of class $C_{\ell}$ in the future. Thus, extinction of class $C_{\ell}$, which we term a partial extinction, corresponds to the event $E_{\ell}=\left\{\right.$ there exists $T>0$ : for all $\left.t \geq T, \boldsymbol{Z}^{\ell}(t)=\mathbf{0}\right\}$. We denote by $\boldsymbol{q}_{k}^{\ell}$ the extinction probability vector of class $C_{\ell}$ given that the initial individual belongs to class $C_{k}$. That is, for each $i \in C_{k}$, the $i$ th entry of $\boldsymbol{q}_{k}^{\ell}$ is given by

$$
\left(\boldsymbol{q}_{k}^{\ell}\right)_{i}=\mathrm{P}\left[E_{\ell} \mid \varphi_{0}=i\right],
$$

and $\boldsymbol{q}^{\ell}=\left[\boldsymbol{q}_{1}^{\ell}, \boldsymbol{q}_{2}^{\ell}, \ldots, \boldsymbol{q}_{m}^{\ell}\right]^{\top}$ denotes the vector of conditional partial extinction probabilities of class $C_{\ell}$, given the class of the initial individual.

The proposition below directly results from the fact that if the whole process $\{\boldsymbol{Z}(t), t \in \mathbb{N}\}$ becomes extinct then all the classes $C_{\ell}$ become extinct too.

Proposition 2.1. It holds that $\boldsymbol{q} \leq \boldsymbol{q}^{\ell}$ for all $1 \leq \ell \leq m$. 


\section{Extinction criteria}

In an irreducible branching process, the extinction probability vector $\boldsymbol{q}$ has either all its components equal to 1 or all its components strictly less than 1 [10]. In a reducible branching process, this dichotomy does not hold anymore: all the components of $\boldsymbol{q}$ are equal to 1 in the subcritical and critical cases, but there might be some components equal to 1 in the supercritical case as well.

Actually, by irreducibility of the equivalence classes, for each $1 \leq k \leq m$, either $\boldsymbol{q}_{k}=\mathbf{1}$ or $\boldsymbol{q}_{k}<\mathbf{1}$, with at least one $k$ such that $\boldsymbol{q}_{k}<\mathbf{1}$ if the whole process is supercritical, and, for each $1 \leq \ell \leq m$, either $\boldsymbol{q}_{k}^{\ell}=\mathbf{1}$ or $\boldsymbol{q}_{k}^{\ell}<\mathbf{1}$. Here, it may thus happen that, starting from one class $C_{k}$, the whole process eventually becomes extinct with probability 1 , so that $\boldsymbol{q}_{k}=\mathbf{1}$, while starting from another class $C_{h}$, the process has a positive probability of surviving, so that $\boldsymbol{q}_{h}<\mathbf{1}$.

We shall assume in the rest of the paper that none of the subprocesses $\left\{\boldsymbol{Z}^{k}(t), t \in \mathbb{N}\right\}$ is singular $(1 \leq k \leq m)$. The first result below provides a necessary and sufficient condition for the total extinction, given the class of the first individual.

Proposition 3.1. For $1 \leq k \leq m$,

$$
\boldsymbol{q}_{k}<\mathbf{1} \Longleftrightarrow \text { there exists } \ell \in \mathscr{D}_{k}: \operatorname{sp}\left(\mathcal{M}_{\ell \ell}\right)>1 \text {. }
$$

Proof. First, we show that the statement

$$
\left.\boldsymbol{q}_{k}<\mathbf{1} \Longleftrightarrow\left(\operatorname{sp}\left(\mathcal{M}_{k k}\right)>1\right) \text { or (there exists } \ell \in \mathcal{E}_{k}: \boldsymbol{q}_{\ell}<\mathbf{1}\right)
$$

implies (3.1). To this end, recall the definition of $\varepsilon_{k}$, and further recursively define

$$
\varepsilon_{k}^{1}=\varepsilon_{k}, \quad \mathcal{E}_{k}^{r}=\bigcup_{\ell \in \mathcal{E}_{k}^{r-1}} \mathcal{E}_{\ell} \quad \text { for } 2 \leq r \leq R(k),
$$

where $R(k)$ is the smallest $r$ such that $\mathcal{E}_{k}^{r}=\varnothing$. With this, if $k \in \mathscr{D}_{k}$ (i.e. if $\mathcal{M}_{k k} \neq 0$ ) then $\mathscr{D}_{k}=\{k\} \cup \bigcup_{r=1}^{R(k)-1} \mathcal{E}_{k}^{r}$, and $\mathscr{D}_{k}=\bigcup_{r=1}^{R(k)-1} \mathcal{E}_{k}^{r}$ otherwise.

Now, expanding the right-hand side of (3.2) we obtain

$$
\begin{aligned}
& \left.\boldsymbol{q}_{k}<\mathbf{1} \Longleftrightarrow\left(\operatorname{sp}\left(\mathcal{M}_{k k}\right)>1\right) \text { or (there exists } \ell \in \mathcal{E}_{k}: \boldsymbol{q}_{\ell}<\mathbf{1}\right) \\
& \Longleftrightarrow \quad\left(\operatorname{sp}\left(\mathcal{M}_{k k}\right)>1\right) \\
& \text { or [there exists } \left.\ell \in \mathcal{E}_{k}:\left(\operatorname{sp}\left(\mathcal{M}_{\ell \ell}\right)>1\right) \text { or (there exists } h \in \mathcal{E}_{\ell}: \boldsymbol{q}_{h}<\mathbf{1}\right) \text { ] } \\
& \left.\Longleftrightarrow\left(\operatorname{sp}\left(\mathcal{M}_{k k}\right)>1\right) \text { or (there exists } \ell \in \mathcal{E}_{k}: \operatorname{sp}\left(\mathcal{M}_{\ell \ell}\right)>1\right) \\
& \text { or (there exists } \ell \in \mathcal{E}_{k}^{2}: \boldsymbol{q}_{\ell}<\mathbf{1} \text { ) } \\
& \left.\Longleftrightarrow\left(\operatorname{sp}\left(\mathcal{M}_{k k}\right)>1\right) \text { or (there exists } \ell \in \mathcal{E}_{k}: \operatorname{sp}\left(\mathcal{M}_{\ell \ell}\right)>1\right) \\
& \text { or (there exists } \left.\ell \in \mathcal{E}_{k}^{2}: \operatorname{sp}\left(\mathcal{M}_{\ell \ell}\right)>1\right) \ldots \\
& \text { or (there exists } \ell \in \mathcal{E}_{k}^{R(k)-1}: \operatorname{sp}\left(\mathcal{M}_{\ell \ell}\right)>1 \text { ) } \\
& \text { or (there exists } \ell \in \mathcal{E}_{k}^{R(k)}: \boldsymbol{q}_{\ell}<\mathbf{1} \text { ) } \\
& \Longleftrightarrow \text { there exists } \ell \in\left(\{k\} \cup \mathcal{E}_{k}^{1} \cup \mathcal{E}_{k}^{2} \cup \cdots \cup \mathcal{E}_{k}^{R(k)-1}\right): \operatorname{sp}\left(\mathcal{M}_{\ell \ell}\right)>1 \\
& \Longleftrightarrow \text { there exists } \ell \in \mathscr{D}_{k}: \operatorname{sp}\left(\mathcal{M}_{\ell \ell}\right)>1 \text {, }
\end{aligned}
$$

yielding (3.1). 
In order to show (3.2), it actually suffices to prove that

$$
\begin{gathered}
\text { there exists } \ell \in \mathcal{E}_{k}: \boldsymbol{q}_{\ell}<\mathbf{1} \quad \Longrightarrow \quad \boldsymbol{q}_{k}<\mathbf{1}, \\
\boldsymbol{q}_{\ell=\mathbf{1}} \quad \text { for all } \ell \in \mathcal{E}_{k} \quad \Longrightarrow \quad\left(\boldsymbol{q}_{k}<\mathbf{1} \Longleftrightarrow \operatorname{sp}\left(\mathcal{M}_{k k}\right)>1\right) .
\end{gathered}
$$

Recall that $\boldsymbol{P}(\boldsymbol{s})$ denotes the offspring generating vector associated to the branching process $\{\boldsymbol{Z}(t), t \in \mathbb{N}\}$; we decompose it as $\boldsymbol{P}(\boldsymbol{s})=\left[\boldsymbol{P}_{1}(\boldsymbol{s}), \boldsymbol{P}_{2}(\boldsymbol{s}), \ldots, \boldsymbol{P}_{m}(\boldsymbol{s})\right]^{\top}$, where the subscript in the subvector $\boldsymbol{P}_{k}(\boldsymbol{s})$ indicates the class of the initial individual, that is, for each $i \in C_{k}$, $\left(\boldsymbol{P}_{k}\right)_{i}(\boldsymbol{s})=\mathrm{E}\left[\boldsymbol{s}^{\boldsymbol{Z}(1)} \mid \varphi_{0}=i\right]$.

By Theorem 2.1, the extinction probability vector $\boldsymbol{q}=\left[\boldsymbol{q}_{1}, \boldsymbol{q}_{2}, \ldots, \boldsymbol{q}_{m}\right]^{\top}$ is the minimal nonnegative solution of the fixed point equation $\boldsymbol{s}=\boldsymbol{P}(\boldsymbol{s})$ and, thus, in particular, for each $1 \leq k \leq m, \boldsymbol{q}_{k}=\boldsymbol{P}_{k}(\boldsymbol{q})$. Assume without loss of generality that $\mathcal{M}_{k k} \neq 0$. Actually, $\boldsymbol{P}_{k}(\boldsymbol{q})$ depends only on $\boldsymbol{q}_{\ell}$ for $\ell=k$ and all $\ell \in \mathcal{E}_{k}$. Let $k_{1}<k_{2}<\cdots<k_{p}$ denote the elements in $\varepsilon_{k}$; we may thus write

$$
\boldsymbol{q}_{k}=\overline{\boldsymbol{P}}_{k}\left(\boldsymbol{q}_{k_{1}}, \boldsymbol{q}_{k_{2}}, \ldots, \boldsymbol{q}_{k_{p}}, \boldsymbol{q}_{k}\right),
$$

where $\overline{\boldsymbol{P}}_{k}\left(\boldsymbol{q}_{k_{1}}, \boldsymbol{q}_{k_{2}}, \ldots, \boldsymbol{q}_{k_{p}}, \boldsymbol{q}_{k}\right)$ is the restriction of $\boldsymbol{P}_{k}(\boldsymbol{q})$ to $\left(\boldsymbol{q}_{j}\right)_{j \in\left(\mathcal{E}_{k} \cup\{k\}\right)}$.

First, assume that $\varepsilon_{k} \neq \varnothing$ and that there exists at least one $\ell \in \boldsymbol{E}_{k}$ such that $\boldsymbol{q}_{\ell}<\mathbf{1}$. Then, $\overline{\boldsymbol{P}}_{k}(\cdot)$ being nondecreasing in each of its variables and strictly increasing in at least one variable of each subvector, we have

$$
\boldsymbol{q}_{k}<\overline{\boldsymbol{P}}_{k}\left(\mathbf{1}, \mathbf{1}, \ldots, \mathbf{1}, \boldsymbol{q}_{k}\right) \leq \mathbf{1}
$$

and we get $\boldsymbol{q}_{k}<\mathbf{1}$, which proves (3.3).

Now, if $\boldsymbol{q}_{\ell}=\mathbf{1}$ for all $\ell \in \varepsilon_{k}$ then

$$
\boldsymbol{q}_{k}=\overline{\boldsymbol{P}}_{k}\left(\mathbf{1}, \mathbf{1}, \ldots, \mathbf{1}, \boldsymbol{q}_{k}\right)=\tilde{\boldsymbol{P}}^{k}\left(\boldsymbol{q}_{k}\right),
$$

where $\tilde{\boldsymbol{P}}^{k}(\cdot)$ is the offspring generating function associated to the irreducible branching process $\left\{\boldsymbol{Z}^{k}(t), t \in \mathbb{N}\right\}$ restricted to class $C_{k}$. The mean offspring matrix corresponding to that branching process is given by $\mathcal{M}_{k k}$, and its total extinction probability vector actually corresponds to $\boldsymbol{q}_{k}^{k}$, that is, the extinction probability of class $C_{k}$, given that the type of the initial individual belongs to this class. By Theorem 2.1, $\boldsymbol{q}_{k}^{k}$ is then the minimal nonnegative solution of $\boldsymbol{s}=\tilde{\boldsymbol{P}}^{k}(\boldsymbol{s})$. Therefore, since $\boldsymbol{q}_{k}$ also satisfies the fixed point equation $\boldsymbol{s}=\tilde{\boldsymbol{P}}^{k}(\boldsymbol{s})$, it must be such that $\boldsymbol{q}_{k} \geq \boldsymbol{q}_{k}^{k}$. On the other hand, by Proposition 2.1, $\boldsymbol{q}_{k} \leq \boldsymbol{q}_{k}^{k}$, so we have $\boldsymbol{q}_{k}=\boldsymbol{q}_{k}^{k}$. Consequently, if $\operatorname{sp}\left(\mathcal{M}_{k k}\right)>1$ then $\left\{\boldsymbol{Z}^{k}(t), t \in \mathbb{N}\right\}$ is supercritical and $\boldsymbol{q}_{k}<\mathbf{1}$; otherwise, $\boldsymbol{q}_{k}=\mathbf{1}$.

If $\varepsilon_{k}=\varnothing$ then the same conclusion holds by an analogous reasoning. Thus, we obtain (3.4).

The second result concerns partial extinction. Starting from an individual in class $C_{k}$, class $C_{\ell}$ survives with positive probability if and only if there exists an intermediate class $C_{h}$ such that $k \rightarrow h \rightarrow \ell$ which is associated to a supercritical subprocess $\left\{\boldsymbol{Z}^{h}(t), t \in \mathbb{N}\right\}$.

Proposition 3.2. For $1 \leq \ell \leq m$ and all $k \in \mathcal{A}_{\ell}$,

$$
\boldsymbol{q}_{k}^{\ell}<\mathbf{1} \Longleftrightarrow \quad \text { there exists } h \in \mathcal{A}_{\ell} \cap \mathscr{D}_{k}: \operatorname{sp}\left(\mathcal{M}_{h h}\right)>1 \text {. }
$$

If $k \notin \mathcal{A}_{\ell}$ then $\boldsymbol{q}_{k}^{\ell}=\mathbf{1}$. 
Proof. If $k \notin \mathcal{A}_{\ell}$ then it is obvious that $\boldsymbol{q}_{k}^{\ell}=\mathbf{1}$ since an individual from class $C_{k}$ will never generate any individual in class $C_{\ell}$. In particular, $\boldsymbol{q}_{k}^{\ell}=\mathbf{1}$ for all $k<\ell$.

Now, let us fix $\ell$. We show by induction on $k$ that, for all $k \in \mathcal{A}_{\ell}$,

$$
\operatorname{sp}\left(\mathcal{M}_{h h}\right) \leq 1 \quad \text { for all } h \in \mathcal{A}_{\ell} \cap \mathscr{D}_{k} \quad \Longrightarrow \quad \boldsymbol{q}_{k}^{\ell}=\mathbf{1} .
$$

Let $\underline{k}=\min _{k}\left\{k \in \mathcal{A}_{\ell}\right\}$. We initiate the induction by showing that if $\operatorname{sp}\left(\mathcal{M}_{h h}\right) \leq 1$ for all $h \in \mathcal{A}_{\ell} \cap \mathscr{D}_{\underline{k}}$ then $\boldsymbol{q}_{k}^{\ell}=\mathbf{1}$.

We denote by $\mathscr{P}^{i}=\left[\mathscr{P}_{1}^{i}, \mathscr{P}_{2}^{i}, \ldots, \mathscr{P}_{m}^{i}\right]$ the vector of direct offspring in each class of an individual of type $i$. For $i \in C_{\underline{k}}$,

$$
\left(\boldsymbol{q}_{\underline{k}}^{\ell}\right)_{i}=\sum_{\boldsymbol{d} \geq \mathbf{0}} \mathrm{P}\left[\mathcal{P}^{i}=\left(\boldsymbol{d}_{1}, \boldsymbol{d}_{2}, \ldots, \boldsymbol{d}_{m}\right)\right] \prod_{1 \leq x \leq m}\left(\boldsymbol{q}_{x}^{\ell}\right)^{\boldsymbol{d}_{x}} .
$$

If $x \in \mathcal{E}_{\underline{k}}$ then $x \notin \mathcal{A}_{\ell}$ by the definition of $\underline{k}$, and, therefore, $\boldsymbol{q}_{x}^{\ell}=\mathbf{1}$. Let $x \neq \underline{k}$. If $x \notin \mathcal{E}_{\underline{k}}$ then $\boldsymbol{d}_{x}=\mathbf{0}$ with probability 1 . So, we find that

$$
\left(\boldsymbol{q}_{\underline{k}}^{\ell}\right)_{i}=\sum_{\boldsymbol{d}_{\underline{k}} \geq \mathbf{0}} \mathrm{P}\left[\mathscr{P}_{\underline{k}}^{i}=\boldsymbol{d}_{\underline{k}}\right]\left(\boldsymbol{q}_{\underline{k}}^{\ell}\right)^{\boldsymbol{d}_{\underline{k}}}=\left(\tilde{\boldsymbol{P}}^{\underline{k}}\right)_{i}\left(\boldsymbol{q}_{\underline{k}}^{\ell}\right),
$$

where recall that $\tilde{\boldsymbol{P}}^{\underline{k}}(\cdot)$ is the offspring generating function of the subprocess $\left\{\boldsymbol{Z}^{\underline{k}}(t), t \in \mathbb{N}\right\}$ restricted to class $C_{\underline{k}}$. Using exactly the same argument as in the proof of Proposition 3.1, we see that $\boldsymbol{q}_{k}^{\ell}$ is a solution of the equation $\boldsymbol{s}=\tilde{\boldsymbol{P}}^{k}(\boldsymbol{s})$, whose minimal nonnegative solution is $\boldsymbol{q}_{k}^{k}$. If $\mathcal{M}_{\underline{k}}=\underline{0}$ then $\boldsymbol{q}_{k}^{\underline{k}}=\mathbf{1}$, and, thus, $\boldsymbol{q}_{k}^{\ell}=\mathbf{1}$. If $\mathcal{M}_{\underline{k}} \neq 0$ then $\underline{k} \in \mathscr{D}_{\underline{k}}$, so that $\mathscr{D}_{\underline{k}} \cap \mathcal{A}_{\ell}=\{\underline{k}\}$; thus, by assumption, $\operatorname{sp}\left(\mathcal{M}_{k k}\right) \leq 1$, and we arrive at the same conclusion.

Now let $K \in \mathcal{A}_{\ell}$ and assume that (3.5) holds for all $k \in \mathcal{A}_{\ell}$ such that $k<K$ (this is our induction assumption). We need to show that it still holds for $K$, that is, if $\operatorname{sp}\left(\mathcal{M}_{h h}\right) \leq 1$ for all $h \in \mathcal{A}_{\ell} \cap \mathscr{D}_{K}$ then $\boldsymbol{q}_{K}^{\ell}=\mathbf{1}$. We can repeat exactly the same argument as for $\underline{k}$ and write (3.6) for $K$, except that now if $x \in \mathcal{E}_{K}$ then $\boldsymbol{q}_{x}^{\ell}=\mathbf{1}$ by the induction assumption because $\mathscr{D}_{x} \subset \mathscr{D}_{K}$.

It remains to show that if there exists a class $C_{h}$ such that $k \rightarrow h \rightarrow \ell$ with $\operatorname{sp}\left(\mathcal{M}_{h h}\right)>1$ then $\boldsymbol{q}_{k}^{\ell}<\mathbf{1}$.

First, since $\operatorname{sp}\left(\mathcal{M}_{h h}\right)>1$, the subprocess $\left\{\boldsymbol{Z}^{h}(t), t \in \mathbb{N}\right\}$ is supercritical and the class $C_{h}$ survives with a positive probability if the initial individual belongs to $C_{h}$. Moreover, since $k \rightarrow h$, each type in $C_{k}$ has a positive probability to generate a type in $C_{h}$. Therefore, it is clear that, starting from a type in $C_{k}$, class $C_{h}$ has a positive probability to survive.

Finally, survival of class $C_{h}$ implies survival of class $C_{\ell}$ since $h \rightarrow \ell$ (see Proposition 3.3(i)). As a consequence, starting from a type in $C_{k}$, class $C_{\ell}$ has a positive probability to survive, that is, $\boldsymbol{q}_{k}^{\ell}<\mathbf{1}$, and the proof is complete.

A direct consequence of Proposition 3.2 is the following.

Corollary 3.1. For $1 \leq \ell \leq m$,

$$
\boldsymbol{q}^{\ell}=\mathbf{1} \Longleftrightarrow \operatorname{sp}\left(\mathcal{M}_{h h}\right) \leq 1 \quad \text { for all } h \in \mathcal{A}_{\ell} .
$$

Now, suppose that we 'observe' the extinction of some class $C_{\ell}$. What can we conclude about the extinction of other classes, on the basis of the structure of $\boldsymbol{M}$ ?

We examine how, by virtue of the relations between classes, knowing the extinction of class $C_{\ell}$ may inform us of the eventual extinction of class $C_{k}$, and even of the whole process. 
For two events $A$ and $B$, we write $A \subseteq B$ if $\mathrm{P}\left[A \cap B^{\mathrm{c}}\right]=0$, and $A \equiv B$ if $A \subseteq B$ and $B \subseteq A$. With this notation, the inclusions $E_{\ell} \subseteq E_{k}$ and $E_{\ell} \subseteq E$ respectively imply that $\boldsymbol{q}^{\ell} \leq \boldsymbol{q}^{k}$ and $\boldsymbol{q}^{\ell} \leq \boldsymbol{q}$, and the equivalences $E_{\ell} \equiv E_{k}$ and $E_{\ell} \equiv E$ respectively imply that $\boldsymbol{q}^{\ell}=\boldsymbol{q}^{k}$ and $\boldsymbol{q}^{\ell}=\boldsymbol{q}$.

We first do not condition on the class of the initial individual, we merely assume that $\mathrm{P}\left[\varphi_{0}=i\right]>0$ for all type $i$, and we give general criteria about the inclusion and equivalence of extinction events.

We show below that if $k \rightarrow \ell$ then, given that class $C_{\ell}$ becomes extinct, class $C_{k}$ almost surely becomes extinct as well. On the other hand, the extinction of class $C_{k}$ implies the extinction of class $C_{\ell}$ if and only if each subprocess $\left\{\boldsymbol{Z}^{h}(t), t \in \mathbb{N}\right\}$ such that $h \rightarrow \ell$ and $h \nrightarrow k$ is subcritical.

Note that in the formulation of the second statement below $k$ must not necessarily belong to $\mathcal{A}_{\ell}$ for $E_{k}$ to imply $E_{\ell}$ (see Remark 3.1 below).

Proposition 3.3. For $1 \leq k, \ell \leq m$,

(i) $k \in \mathcal{A}_{\ell}$ implies that $E_{\ell} \subseteq E_{k}$,

(ii) $\operatorname{sp}\left(\mathcal{M}_{h h}\right) \leq 1$ for all $h \in \mathcal{A}_{\ell} \backslash \mathcal{A}_{k}$ if and only if $E_{k} \subseteq E_{\ell}$.

Statements ( $i$ ) and (ii) actually provide the following sufficient condition for the equivalence of extinction events: for $k \in \mathcal{A}_{\ell}$ and all $h \in \mathcal{A}_{\ell} \backslash \mathcal{A}_{k}$,

$$
\operatorname{sp}\left(\mathcal{M}_{h h}\right) \leq 1 \quad \Longrightarrow \quad E_{k} \equiv E_{\ell}
$$

Proof. (i) Suppose that $k \in \mathcal{A}_{\ell}$. We have to show that $E_{\ell} \subseteq E_{k}$, or, equivalently, that $E_{k}^{\mathrm{c}} \subseteq E_{\ell}^{\mathrm{c}}$. Let $V_{\ell}$ be the total number of individuals born in class $C_{\ell}$. Then, $E_{\ell}^{\mathrm{c}} \equiv\left[V_{\ell}=\infty\right]$. Let us show that $E_{k}^{\mathrm{c}} \subseteq\left[V_{\ell}=\infty\right]$.

Let $t_{1} \leq t_{2} \leq t_{3} \leq \cdots$ be the successive birth times of individuals in $C_{k}$. As we assume the survival of class $C_{k}$, this sequence is almost surely infinite. Now, let $X_{n}$ denote the indicator of the event $\left\{\right.$ there exists $\left.t>t_{n}: \boldsymbol{Z}^{\ell}(t) \neq \mathbf{0}\right\}$. The assumption that $k \in \mathcal{A}_{\ell}$ implies that

$$
\mathrm{P}\left[X_{n}=1 \mid Z^{k}\left(t_{n}\right) \neq \mathbf{0}\right]>0
$$

for all $n \geq 1$. Therefore, $V_{\ell} \geq \sum_{1 \leq n \leq \infty} X_{n}=\infty$ almost surely, which proves that $E_{k}^{\mathrm{c}} \subseteq E_{\ell}^{\mathrm{c}}$.

(ii) Now, assume that, for all $h \in \mathcal{A}_{\ell} \backslash \mathcal{A}_{k}, \operatorname{sp}\left(\mathcal{M}_{h h}\right) \leq 1$. We have to show that $E_{k} \subseteq E_{\ell}$, that is, $E_{\ell}^{\mathrm{c}} \cap E_{k} \equiv \varnothing$.

Note that if $\ell \in \mathcal{A}_{k}$ then $\mathcal{A}_{\ell} \backslash \mathcal{A}_{k}=\varnothing$ and, from (i), $E_{k} \subseteq E_{\ell}$. Let us assume from now on that $\ell \notin \mathcal{A}_{k}$, and that both events $E_{\ell}^{\mathrm{c}}$ and $E_{k}$ occur simultaneously with positive probability. We have to show that we obtain a contradiction.

We either have $\mathcal{M}_{\ell \ell}=0$ or $\ell \in \mathcal{A}_{\ell} \backslash \mathcal{A}_{k}$, so that, by assumption, $\operatorname{sp}\left(\mathcal{M}_{\ell \ell}\right) \leq 1$. As a consequence, since, by assumption, $C_{\ell}$ survives and $C_{k}$ becomes extinct with positive probability, $\overline{\mathscr{A}}_{\ell} \backslash \mathcal{A}_{k} \neq \varnothing$, where $\overline{\mathcal{A}}_{\ell}=\mathcal{A}_{\ell} \backslash\{\ell\}$, and there is at least one $h \in \overline{\mathcal{A}}_{\ell} \backslash \mathcal{A}_{k}$ such that $V_{h}=\infty$ almost surely. Let

$$
\bar{h}=\max _{h}\left\{h \in \overline{\mathcal{A}}_{\ell} \backslash \mathcal{A}_{k}: V_{h}=\infty\right\} .
$$

Then $V_{\bar{h}}=\infty$ and $V_{x}<\infty$ almost surely for all $x \in \overline{\mathcal{A}}_{\bar{h}} \subseteq \mathcal{A}_{\ell}$. This implies that $\operatorname{sp}\left(\mathcal{M}_{\bar{h} \bar{h}}\right)>1$, which contradicts the assumptions. We thus have $E_{\ell}^{\mathrm{c}} \cap E_{k} \equiv \varnothing$. 
Finally, let us show that, if there exists $h \in \mathcal{A}_{\ell} \backslash \mathcal{A}_{k} \operatorname{such}$ that $\operatorname{sp}\left(\mathcal{M}_{h h}\right)>1, \mathrm{P}\left[E_{k} \cap E_{\ell}^{\mathrm{c}}\right]>0$. Let us fix $h$ in $\mathcal{A}_{\ell} \backslash \mathcal{A}_{k}$ such that $\operatorname{sp}\left(\mathcal{M}_{h h}\right)>1$. We have, for any $i \in C_{h}$,

$$
\mathrm{P}\left[E_{k} \cap E_{\ell}^{\mathrm{c}}\right] \geq \mathrm{P}\left[\varphi_{0}=i\right] \mathrm{P}\left[E_{k} \cap E_{\ell}^{\mathrm{c}} \mid \varphi_{0}=i\right] .
$$

The second factor on the right-hand side of (3.7) is such that

$$
\mathrm{P}\left[E_{k} \cap E_{\ell}^{\mathrm{c}} \mid \varphi_{0}=i\right] \geq \mathrm{P}\left[E_{h}^{\mathrm{c}} \mid \varphi_{0}=i\right] \mathrm{P}\left[E_{k} \cap E_{\ell}^{\mathrm{c}} \mid \varphi_{0}=i, E_{h}^{\mathrm{c}}\right] .
$$

But, by assumption, $\operatorname{sp}\left(\mathcal{M}_{h h}\right)>1$; thus, $\mathrm{P}\left[E_{h}^{\mathrm{c}} \mid \varphi_{0}=i\right]>0$, and, as $h \notin \mathcal{A}_{k}, \mathrm{P}\left[E_{k} \mid \varphi_{0}=i\right.$, $\left.E_{h}^{\mathrm{c}}\right]=1$, so that

$$
\mathrm{P}\left[E_{k} \cap E_{\ell}^{\mathrm{c}} \mid \varphi_{0}=i, E_{h}^{\mathrm{c}}\right]=\mathrm{P}\left[E_{\ell}^{\mathrm{c}} \mid \varphi_{0}=i, E_{h}^{\mathrm{c}}\right] .
$$

Now, since $h \in \mathcal{A}_{\ell}$, by (i) we know that $\mathrm{P}\left[E_{\ell}^{\mathrm{c}} \mid E_{h}^{\mathrm{c}}\right]=1$, so $\mathrm{P}\left[E_{\ell}^{\mathrm{c}} \mid \varphi_{0}=i, E_{h}^{\mathrm{c}}\right]>0$, and the right-hand side of (3.8) is strictly positive, which implies that the right-hand side of (3.7) is strictly positive as well, and, thus, $\mathrm{P}\left[E_{k} \cap E_{\ell}^{\mathrm{c}}\right]>0$.

The extinction of class $C_{\ell}$ is equivalent to the extinction of the whole process if all classes $C_{k}$ such that $k \nrightarrow \ell$ eventually become extinct with probability 1 , or if $k \rightarrow \ell$ for all $k$ (and, thus, $\ell=1)$.

Proposition 3.4. The following sufficient conditions hold for the extinction of a class to be equivalent to the total extinction.

(i) For $1 \leq \ell \leq m$, if, for all $k \notin \mathcal{A}_{\ell}, \boldsymbol{q}^{k}=\mathbf{1}$ then $E_{\ell} \equiv E$.

(ii) If $\mathcal{A}_{1}=\{1,2, \ldots, m\}$ then $E_{1} \equiv E$.

Proof. We already know from Proposition 2.1 that $E \subseteq E_{\ell}$ for all $1 \leq \ell \leq m$.

(i) If $k \in \mathcal{A}_{\ell}$ then, by Proposition 3.3(i), $E_{\ell} \subseteq E_{k}$. If $\bar{k} \notin \mathcal{A}_{\ell}$ then, by assumption, $\boldsymbol{q}^{k}=\mathbf{1}$, which implies that $\mathrm{P}\left[E_{k}^{\mathrm{c}}\right]=0$, so that $\mathrm{P}\left[E_{\ell} \cap E_{k}^{\mathrm{c}}\right]=0$, and $E_{\ell} \subseteq E_{k}$.

We thus have $E_{\ell} \subseteq E_{k}$ for all $1 \leq k \leq m$, and, therefore, $E_{\ell} \subseteq E$, which implies that $E_{\ell} \equiv E$.

(ii) If $\mathcal{A}_{1}=\{1,2, \ldots, m\}$, we know from Proposition 3.3(i) that $E_{1} \subseteq E_{k}$ for all $1 \leq k \leq m$, so that $E_{1} \subseteq E$, and, thus, $E_{1} \equiv E$.

Remark 3.1. Propositions 3.3(i) and 3.4 only give sufficient conditions. Let us show with an example that they are not necessary conditions. Take the mean offspring matrix

$$
\boldsymbol{M}=\left[\begin{array}{ccc}
\mathcal{M}_{11} & 0 & 0 \\
0 & \mathcal{M}_{22} & 0 \\
\mathcal{M}_{31} & \mathcal{M}_{32} & \mathcal{M}_{33}
\end{array}\right],
$$

and assume that $\operatorname{sp}\left(\mathcal{M}_{22}\right) \leq 1$ and $\operatorname{sp}\left(\mathcal{M}_{33}\right)>1$. By definition, $\mathcal{A}_{1}=\{1,3\}, \mathcal{A}_{2}=\{2,3\}$, and $\mathcal{A}_{3}=\{3\}$. We have $2 \notin \mathcal{A}_{1}$, but $E_{1} \subseteq E_{2}$ since $E_{1} \subseteq E_{3}$ by Proposition 3.3(i), and $E_{3} \subseteq E_{2}$ by Proposition 3.3(ii). Thus, we may have $E_{\ell} \subseteq E_{k}$ with $\ell<k$, even if $k$ is not in $\mathcal{A}_{\ell}$.

Furthermore, $E_{1} \equiv E$, but $2 \notin \mathcal{A}_{1}$ and $\boldsymbol{q}^{2} \neq \mathbf{1}$, since $\boldsymbol{q}_{3}^{2}<\mathbf{1}$ by Proposition 3.2. Thus, Proposition 3.4 does not provide necessary conditions either.

Note that if, in addition, $\operatorname{sp}\left(\mathcal{M}_{11}\right) \leq 1$ then, by Proposition 3.3(ii), $E_{2} \subseteq E_{1}$, even if $2 \notin \mathcal{A}_{1}$ and $E_{3} \subseteq E$ by Proposition 3.4. Thus, $E_{1} \equiv E_{2} \equiv E_{3} \equiv E$ in that case.

Sometimes, the effect of the extinction of a class on another class depends on the type of the initial individual; if we know that it belongs to some class $C_{h}$ then the assumptions of Propositions 3.3 and 3.4 must be verified for the classes in $\mathscr{D}_{h}$ only. 
Corollary 3.2. For all $1 \leq \ell, k, h \leq m$,

(i) if $C_{\ell}, C_{k}$, and $C_{h}$ are three classes such that

- $k \in \mathcal{A}_{\ell}$,

- $\left(\mathcal{A}_{\ell} \backslash \mathcal{A}_{k}\right) \cap \mathscr{D}_{h} \neq \varnothing$, and

- $\operatorname{sp}\left(\mathcal{M}_{x x}\right) \leq 1$ for all $x \in\left(\mathcal{A}_{\ell} \backslash \mathcal{A}_{k}\right) \cap \mathscr{D}_{h}$,

then $E_{k} \cap\left[\varphi_{0}=i\right] \equiv E_{\ell} \cap\left[\varphi_{0}=i\right]$ for all $i \in C_{h}$,

(ii) if $\mathscr{D}_{h} \subseteq \mathcal{A}_{\ell}$ or, for all $x \in \mathcal{D}_{h} \backslash \mathcal{A}_{\ell}, \boldsymbol{q}_{h}^{x}=\mathbf{1}$, then $E_{\ell} \cap\left[\varphi_{0}=i\right] \equiv E \cap\left[\varphi_{0}=i\right]$ for all $i \in C_{h}$.

The proof is very similar to those of Propositions 3.3 and 3.4, and is thus omitted.

\section{Partial extinction}

Now that we have established necessary and sufficient criteria for the almost-sure extinction of classes, we characterize the vector $\boldsymbol{q}^{\ell}$ of extinction probabilities of class $C_{\ell}$ as one particular solution of a fixed point equation.

Theorem 2.2 asserts that in the irreducible case, for any $\mathbf{0} \leq \boldsymbol{a} \leq \mathbf{1}$ with $\boldsymbol{a} \neq \mathbf{1}$, we have

$$
\lim _{n \rightarrow \infty} \boldsymbol{P}^{(n)}(\boldsymbol{a})=\boldsymbol{q},
$$

and, as a consequence, the only solutions of the extinction equation $\boldsymbol{s}=\boldsymbol{P}(\boldsymbol{s})$ in the unit cube are $\mathbf{1}$ and $\boldsymbol{q}$ (see [5, Chapter 2, Corollary 1]). In the reducible case, this is no longer the case, each $\boldsymbol{q}^{\ell}$ is also a solution of the extinction equation, which may thus have up to $m+1$ distinct solutions. The limit of $\boldsymbol{P}^{(n)}(\boldsymbol{a})$ when $n$ tends to $\infty$ actually depends on the vector $\boldsymbol{a}$.

Theorem 4.1. For each $1 \leq \ell \leq m$, the vector of extinction probabilities $\boldsymbol{q}^{\ell}$ of class $C_{\ell}$ is the smallest nonnegative solution of the extinction equation $\boldsymbol{s}=\boldsymbol{P}(\boldsymbol{s})$ such that $\boldsymbol{q}_{k}^{\ell}=\mathbf{1}$ for each $k \nrightarrow \ell$.

Proof. We follow the same arguments as in [10, Theorem 7.1]. Let $\boldsymbol{q}^{\ell}(n)$ denote the probability that all classes in $\mathcal{A}_{\ell}$ become extinct no later than the $n$th generation. We have $\boldsymbol{q}^{\ell}(n) \leq \boldsymbol{q}^{\ell}(n+1)$ and $\boldsymbol{q}^{\ell}=\lim _{n \rightarrow \infty} \boldsymbol{q}^{\ell}(n)$. The probabilities $\boldsymbol{q}^{\ell}(n)$ may be computed recursively, i.e.

$$
\boldsymbol{q}^{\ell}(1)=\boldsymbol{P}\left(f^{\ell}\right),
$$

where $\boldsymbol{f}^{\ell}$ is the indicator vector of the classes which do not lead to class $C_{\ell}$, that is, $\boldsymbol{f}_{k}^{\ell}=\mathbf{1}$ for $k \nrightarrow \ell$, and $\boldsymbol{f}_{k}^{\ell}=\mathbf{0}$ for $k \rightarrow \ell$. Indeed, for all classes in $\mathcal{A}_{\ell}$ to become extinct at the first generation, the initial individual must not produce any individual in a class belonging to $\mathcal{A}_{\ell}$. For $n \geq 2$,

$$
\boldsymbol{q}^{\ell}(n)=\boldsymbol{P}\left(\boldsymbol{q}^{\ell}(n-1)\right) .
$$

Since the sequence $\left\{\boldsymbol{q}^{\ell}(n)\right\}_{n \geq 1}$ is nondecreasing in $n$ and the generating functions are continuous in each variable, we obtain

$$
\boldsymbol{q}^{\ell}=\boldsymbol{P}\left(\boldsymbol{q}^{\ell}\right)
$$

which shows that $\boldsymbol{q}^{\ell}$ is a solution of $s=\boldsymbol{P}(\boldsymbol{s})$ with the property that $\boldsymbol{q}_{k}^{\ell}=\mathbf{1}$ for each $k \nrightarrow \ell$. 
It remains to show that $\boldsymbol{q}^{\ell}$ is the minimal nonnegative solution of $\boldsymbol{s}=\boldsymbol{P}(\boldsymbol{s})$ under the specific constraint $\boldsymbol{q}_{k}^{\ell}=\mathbf{1}$ for each $k \nrightarrow \ell$. Let $\boldsymbol{q}^{*}$ be any other such vector. We have $\boldsymbol{q}^{*} \geq \boldsymbol{f}^{\ell}$, and, thus,

$$
\boldsymbol{q}^{*}=\boldsymbol{P}\left(\boldsymbol{q}^{*}\right) \geq \boldsymbol{P}\left(\boldsymbol{f}^{\ell}\right)=\boldsymbol{q}^{\ell}(1),
$$

by the monotonicity of $\boldsymbol{P}(\cdot)$, and we finally get $\boldsymbol{q}^{*} \geq \boldsymbol{P}\left(\boldsymbol{q}^{\ell}(n)\right)$ for all $n \geq 1$. Then, letting $n$ tend to $\infty$, we obtain $\boldsymbol{q}^{*} \geq \boldsymbol{q}^{\ell}$, completing the proof.

This shows again that $\boldsymbol{q} \leq \boldsymbol{q}^{\ell}$ for all $1 \leq \ell \leq m$, since $\boldsymbol{q}$ is the minimal nonnegative solution of $\boldsymbol{s}=\boldsymbol{P}(\boldsymbol{s})$ without any constraint.

Theorem 4.1 opens the way for algorithmic developments, as we show in the sequel, but before doing so, we observe that it may be interpreted as follows: the extinction probability vector $\boldsymbol{q}^{\ell}$ of class $C_{\ell}$ also corresponds to the total extinction probability vector $\hat{\boldsymbol{q}}$ of a modified branching process $\{\hat{\boldsymbol{Z}}(t), t \in \mathbb{N}\}$, in which we fix at 0 the birth rate of an individual which is not able to generate individuals in class $C_{\ell}$. In terms of processes, we thus have the following property.

Proposition 4.1. The extinction probability vector $\boldsymbol{q}^{\ell}$ is the minimal nonnegative solution of the extinction equation $\boldsymbol{s}=\hat{\boldsymbol{P}}(\boldsymbol{s})$, where $\hat{\boldsymbol{P}}(\boldsymbol{s})$ is the offspring generating function of the modified branching process $\{\hat{\boldsymbol{Z}}(t), t \in \mathbb{N}\}$, in which we set $\hat{\boldsymbol{P}}_{k}(\boldsymbol{s}):=\mathbf{1}$ for all $k \nrightarrow \ell$.

Proof. We have

$$
\begin{aligned}
\boldsymbol{q}^{\ell} & =\mathrm{P}\left[\text { there exists } T_{1}>0: \boldsymbol{Z}_{k}\left(T_{1}\right)=\mathbf{0} \text { for all } k \rightarrow \ell \mid \varphi_{0}\right] \\
& =\mathrm{P}\left[\text { there exists } T_{1}>0: \hat{\boldsymbol{Z}}_{k}\left(T_{1}\right)=\mathbf{0} \text { for all } k \rightarrow \ell \mid \varphi_{0}\right],
\end{aligned}
$$

and, as the individuals unable to generate individuals in class $C_{\ell}$ eventually die without any offspring in the modified branching process,

$$
\begin{aligned}
& \mathrm{P}\left[\text { there exists } T_{1}>0: \hat{\mathbf{Z}}_{k}\left(T_{1}\right)=\mathbf{0} \text { for all } k \rightarrow \ell \mid \varphi_{0}\right] \\
& =\mathrm{P}\left[\text { there exists } T_{2} \geq T_{1}: \hat{\boldsymbol{Z}}_{i} k\left(T_{2}\right)=\mathbf{0} \text { for all } k \rightarrow \ell\right. \\
& \left.\quad \quad \text { and } \hat{\boldsymbol{Z}}_{k}\left(T_{2}\right)=\mathbf{0} \text { for all } k \nrightarrow \ell \mid \varphi_{0}\right] \\
& =\mathrm{P}\left[\text { there exists } T_{2}>0: \hat{\mathbf{Z}}\left(T_{2}\right)=\mathbf{0} \mid \varphi_{0}\right] \\
& =\hat{\boldsymbol{q}} .
\end{aligned}
$$

This shows that $\boldsymbol{q}^{\ell}=\hat{\boldsymbol{q}}$.

The extinction criteria of a class $C_{\ell}$ are thus equivalent to the total extinction criteria of the modified branching process (still reducible).

One can interpret these two results in light of Sewastjanow [13, Chapter 4, pp. 100-101]. Defining a regular set of types $S$ as a set such that if $i, j \in S$, where type $i \rightarrow k$, and $k \rightarrow j$ for some intermediate type $k$, then $k \in S$, Sewastjanow showed that the process obtained by restricting the branching process to types belonging to a regular set $S$ is a new branching process. In our case, the classes belonging to $\mathcal{A}_{\ell}$ form a regular set of types, and we observe that the extinction of class $C_{\ell}$ is equivalent to the extinction of the set of types in $\mathcal{A}_{\ell}$, that is, $E_{\ell} \equiv\left\{\bigcap_{k \in \mathcal{A}_{\ell}} E_{k}\right\}$. Indeed, we already know from Proposition 3.3 that $E_{\ell} \subseteq E_{k}$ for all $k \in \mathcal{A}_{\ell}$, and it is clear that the extinction of all ancestors of $C_{\ell}$ implies the extinction of $C_{\ell}$.

In the rest of the paper we discuss computational issues. In order to prove the next result, we need the following dichotomy property: assuming that there is a path to extinction from each 
type of individual, that is, $q_{i}>0$ for all $1 \leq i \leq n$, a nonsingular branching process $\{\boldsymbol{Z}(t)$, $t \in \mathbb{N}$ \} either grows to $\infty$, or goes to $\mathbf{0}$ :

$$
\mathrm{P}\left[\lim _{t \rightarrow \infty}|\boldsymbol{Z}(t)|=+\infty\right]+\mathrm{P}\left[\lim _{t \rightarrow \infty}|\boldsymbol{Z}(t)|=0\right]=1 .
$$

It does not remain positive and bounded [5, Chapter 2, Theorem 6.1].

The counterpart of Theorem 2.2 in the present case is then provided by the following theorem.

Theorem 4.2. For all $1 \leq \ell \leq m$ and every vector $\boldsymbol{a}^{(\ell)}$ such that

$$
\boldsymbol{a}_{k}^{(\ell)}\left\{\begin{array}{l}
=\mathbf{1} \text { for all } k \nrightarrow \ell, \\
<\mathbf{1} \text { for all } k \rightarrow \ell,
\end{array}\right.
$$

we have

$$
\lim _{n \rightarrow \infty} \boldsymbol{P}^{(n)}\left(\boldsymbol{a}^{(\ell)}\right)=\boldsymbol{q}^{\ell}
$$

Proof. Let us fix $\ell$. For clarity of exposition, we reorder the $n$ types of the branching process into two classes: $L_{\bullet}=\bigcup_{k \in \mathcal{A}_{\ell}} C_{k}$ and $L_{\circ}=\{1,2, \ldots, n\} \backslash L_{\bullet}$. Hence, we may rewrite $\boldsymbol{a}^{(\ell)}=\left[\boldsymbol{a}_{\bullet}, \boldsymbol{a}_{\circ}\right]^{\top}$ with $\boldsymbol{a}_{\bullet}<\mathbf{1}$ and $\boldsymbol{a}_{\circ}=\mathbf{1}$.

As in the proof of Theorem 4.1, we consider successive generations and we define $\boldsymbol{Z}_{n}$ to be the population size at the $n$th generation. We have, for any finite $N \in \mathbb{N}_{0}$,

$$
\begin{aligned}
\boldsymbol{P}^{(n)}\left(\boldsymbol{a}^{(\ell)}\right)= & \sum_{\boldsymbol{y} \geq \mathbf{0}} \mathrm{P}\left[\boldsymbol{Z}_{n}=(\mathbf{0}, \boldsymbol{y}) \mid \varphi_{0}\right]+\sum_{\substack{\boldsymbol{y} \geq \mathbf{0} \\
\boldsymbol{x} \geq \mathbf{0}, \boldsymbol{x} \neq \mathbf{0} \\
\|\boldsymbol{x}\| \leq N}} \mathrm{P}\left[\boldsymbol{Z}_{n}=(\boldsymbol{x}, \boldsymbol{y}) \mid \varphi_{0}\right] \boldsymbol{a}_{\bullet}^{\boldsymbol{x}} \\
& +\sum_{\substack{\boldsymbol{y} \geq \mathbf{0} \\
\boldsymbol{x} \geq \mathbf{0}, \boldsymbol{x} \neq \mathbf{0} \\
\|\boldsymbol{x}\|>N}} \mathrm{P}\left[\boldsymbol{Z}_{n}=(\boldsymbol{x}, \boldsymbol{y}) \mid \varphi_{0}\right] \boldsymbol{a}_{\bullet}^{\boldsymbol{x}} \cdot
\end{aligned}
$$

The first term approaches $\boldsymbol{q}^{\ell}$ as $n$ tends to $\infty$. The second term tends to 0 as $n$ tends to $\infty$. Indeed, for all $i \in L_{\circ}$,

$$
\sum_{\substack{\boldsymbol{y} \geq \mathbf{0} \\ \boldsymbol{x} \geq \mathbf{0}, \boldsymbol{x} \neq \mathbf{0} \\\|\boldsymbol{x}\| \leq N}} \mathrm{P}\left[\boldsymbol{Z}_{n}=(\boldsymbol{x}, \boldsymbol{y}) \mid \varphi_{0}=i\right]=0,
$$

since if the initial individual belongs to $L_{\circ}$ then it is unable to generate an individual in $C_{\ell}$, which implies that it is not able to generate an individual in $L_{\bullet}$ either. For all $i \in L_{\bullet}$,

$$
\lim _{n \rightarrow \infty} \sum_{\substack{\boldsymbol{y} \geq \mathbf{0} \\ \boldsymbol{x} \geq \mathbf{0}, \boldsymbol{x} \neq \mathbf{0} \\\|\boldsymbol{x}\| \leq N}} \mathrm{P}\left[\boldsymbol{Z}_{n}=(\boldsymbol{x}, \boldsymbol{y}) \mid \varphi_{0}=i\right]=0,
$$

since if the first individual belongs to $L_{\bullet}$ then $\left(\boldsymbol{Z}_{n}\right)_{\bullet}$ is the restriction of the branching process limited to the types in $L_{\bullet}$, which is another branching process, itself exploding or becoming extinct by the dichotomy property stated in (4.1). In the limit, $\|x\|$ can thus not be both nonzero and less than or equal to $N$.

Finally, the third term tends to 0 since it is dominated by $\mathrm{P}\left[\left\|\boldsymbol{Z}_{n}\right\|>N \mid \varphi_{0}\right]$ times the $N$ th power of a quantity strictly less than 1 , and $N$ is arbitrary. 
Theorems 4.1 and 4.2 suggest a method to numerically compute $\boldsymbol{q}^{\ell}$ for each class $C_{\ell}$ in a reducible multitype branching process: starting with the initial vector $\boldsymbol{x}^{\ell}(0)=\boldsymbol{a}^{(\ell)}$ such that

$$
\boldsymbol{a}_{k}^{(\ell)}= \begin{cases}\mathbf{1} & \text { for all } k \nrightarrow \ell, \\ \boldsymbol{P}_{k}(\mathbf{0})<\mathbf{1} & \text { for all } k \rightarrow \ell,\end{cases}
$$

the functional iteration $\boldsymbol{x}^{\ell}(n)=\boldsymbol{P}\left(\boldsymbol{x}^{\ell}(n-1)\right), n \geq 1$, converges to the vector $\boldsymbol{q}^{\ell}$.

Another more efficient way to compute partial extinction probabilities would be to use Proposition 4.1 and to compute $\boldsymbol{q}^{\ell}$ as the total extinction probability of the branching process modified such that $\hat{\boldsymbol{P}}_{k}(\boldsymbol{s})=\mathbf{1}$ for all $k \nrightarrow \ell$. This allows for the direct use of any available algorithm. For instance, in some special cases of multitype branching processes called Markovian trees, several linear and quadratic algorithms have been developed to compute the total extinction probability; see [1], [6], and [7].

\section{Acknowledgements}

This work was conducted when the author was a research fellow of the Fonds National de la Recherche Scientifique (F.R.S. - F.N.R.S.). It comprises a part of her doctoral thesis supervised by Professor Guy Latouche; she would like to thank him for his helpful comments and suggestions. The author also acknowledges the support of the Australian Research Council, grant number DP110101663.

\section{References}

[1] Bean, N. G., Kontoleon, N. and Taylor, P. G. (2008). Markovian trees: properties and algorithms. Ann. Operat. Res. 160, 31-50.

[2] Foster, J. AND Ney, P. (1976). Decomposable critical multi-type branching processes. Sankhyā A 38, 28-37.

[3] Foster, J. ANd Ney, P. (1978). Limit laws for decomposable critical branching processes. Z. Wahrscheinlichkeitsth. 46, 13-43.

[4] Gantmacher, F. R. (1974). The Theory of Matrices. Chelsa Publishing, New York.

[5] Harris, T. E. (1963). The Theory of Branching Processes. Springer, Berlin.

[6] Hautphenne, S., Latouche, G. and Remiche, M.-A. (2008). Newton's iteration for the extinction probability of a Markovian binary tree. Linear Algebra Appl. 428, 2791-2804.

[7] Hautphenne, S., Latouche, G. and Remiche, M.-A. (2011). Algorithmic approach to the extinction probability of branching processes. Methodology Comput. Appl. Prob. 13, 171-192.

[8] Kesten, H. and Stigum, B. P. (1966). A limit theorem for multidimensional Galton-Watson processes. Ann. Math. Statist. 37, 1211-1223.

[9] Kesten, H. And Stigum, B. P. (1967). Limit theorems for decomposable multi-dimensional Galton-Watson processes. J. Math. Anal. Appl. 17, 309-338.

[10] Mode, C. J. (1971). Multitype Branching Processes. Theory and Applications. Elsevier, New York.

[11] Olofsson, P. (2000). A branching process model of telomere shortening. Commun. Statist. Stoch. Models 16, 167-177.

[12] Scalia-Tomba, G.-P. (1986). The asymptotic final size distribution of reducible multitype Reed-Frost processes. J. Math. Biol. 23, 381-392.

[13] Sewastuanow, B. A. (1975). Verzweigungsprozesse. R. Oldenbourg, Munich.

[14] Sugitani, S. (1979). On the limit distribution of decomposable Galton-Watson processes. Proc. Japan Acad. $\mathbf{5 5}, 334-336$. 